\title{
Pion constituent quark couplings strong form factors: A dynamical approach
}

\author{
Fábio L. Braghin \\ Instituto de Física, Federal University of Goias, Av. Esperança, s/n, 74690-900 Goiânia, GO, Brazil
}

(Received 24 July 2018; published 2 January 2019)

\begin{abstract}
Form factors for pion interactions with constituent quarks are investigated as the leading effective couplings obtained from a one-loop background field method applied to a global color model. Two pion field definitions are considered and the resulting eleven form factors are expressed in terms of components of the quark and gluon propagators that compose only two momentum-dependent functions. A momentumdependent Goldberger-Treiman relation is also obtained as one of the ratios between the form factors. The resulting form factors with pion momenta up to $1.5 \mathrm{GeV}$ are exhibited for different quark effective masses and two different nonperturbative gluon propagators and they present similar behavior to fittings of experimental data from nucleons form factors. The corresponding pseudoscalar averaged quadratic radii (a.q.r.) and correction to the axial a.q.r. are presented as functions of the sea quark effective mass, being equal, respectively, to the scalar and vector ones at the present level of calculation.
\end{abstract}

DOI: 10.1103/PhysRevD.99.014001

\section{INTRODUCTION}

The strong, electromagnetic and weak content of hadrons has been under continuous intense theoretical and experimental scrutiny. Different hadron form factors are among the main observables for understanding details of their interactions and structures, including size, and they are important quantities to compare theoretical and experimental results [1-5]. For example, the vector form factors provide the charge and electromagnetic hadron structure and interactions, the nucleon axial form factor provides important information for their spin structure and weak interaction observables such as neutron beta decay or CKM matrix unitarity. There are many theoretical calculations for the light hadrons strong form factors, for example [6-18] and references therein. Lately, lattice estimations for pionnucleon/baryons interactions were provided for progressively lower values of the pion mass, for example in $[10,17,18]$. Concerning their very low momentum behavior, experimental results for nucleon electromagnetic and strong averaged radii provide values $\sqrt{\left\langle r^{2}\right\rangle} \simeq 0.8-0.9 \mathrm{fm}$ $[1,13,19,20]$.

In spite of the many difficulties to provide a complete description of hadrons and their interactions compatible with experimental data directly from QCD, in particular in

Published by the American Physical Society under the terms of the Creative Commons Attribution 4.0 International license. Further distribution of this work must maintain attribution to the author(s) and the published article's title, journal citation, and DOI. Funded by SCOAP . the low and intermediary energies regimes, both effective models and effective theories have been considered to understand partial or isolated aspects of strong interactions. Among these models the constituent quark models (CQM) has shown to describe many aspects of hadron structure and interactions by considering dressed quark degrees of freedom (d.o.f.), dynamical chiral symmetry breaking (DChSB) and eventually a pion cloud, [12,21-23]. Within the constituent quark model it has been argued that the zero momentum limit of the axial form factor should be $g_{A}(0)=3 / 4$ or $g_{A}(0)=1[12,21]$. Also, a radius of the order of $0.2-0.3 \mathrm{fm}$ has been estimated for constituent quarks [12,24]. In the Weinberg's large Nc effective field theory (EFT), constituent quarks and gluons interact with pions whose dynamics is ruled by the leading terms of chiral perturbation theory (ChPT), coping with the large Nc expansion [21]. In [25,26], this EFT has been derived as the leading terms from a large quark and gluon effective masses expansion for the one-loop background field method applied to a global color model in the vacuum and with leading couplings to the electromagnetic field. It can be expected that, by comparing the strong and electromagnetic nucleon and light mesons form factors with those for constituent quarks, the detailed role and contribution of each internal d.o.f. for the details of hadron structure and interactions might be elucidated clearly. Of course, to accomplish this program, besides further comparisons between different theoretical frameworks, it is also important to improve the amount and precision of experimental data. This means that the related developments might shed light on the partial or even complete reliability of CQMtype models to describe hadron interactions in particular 
energy ranges. Moreover, these comparisons might make explicit particular effects or mechanisms present in hadron structure and interactions by means of analytical or semianalytical approaches besides well-established lattice QCD framework. Eventually, it can be used to assess or to improve field theoretic schemes for an eventual unambiguous parametrization of the nucleon and nuclear potentials [27].

In the present work, the strong constituent quark form factors associated to the leading pion couplings to constituent quarks are derived and investigated. This method was considered before for the zero momentum limit of the corresponding pion-constituent quark couplings [25,26] and for the light vector mesons momentum-dependent couplings to constituent quarks [28,29]. The form factors are obtained from a large quark and gluon effective masses expansion for the one-loop background field applied to a global color model. The background field quark becomes the constituent quark due to the one-loop calculation in which an internal (nonperturbative) gluon line dresses the (background) quark. This is nearly independent from the dynamical symmetry breaking, except for the fact that the same gluon propagator required to yield DChSB is considered. This momentum-dependent constituent quark mass emerges, therefore, by means of a different mechanism from the usual DChSB. This might be in agreement with recent calculations [30]. The resulting couplings and form factors, therefore, correspond to tree-level pionconstituent quark vertices. These pion-constituent quarks form factors are investigated and comparisons with experimental data for pion nucleon are presented. Furthermore, four further pion derivative couplings with scalar and pseudoscalar constituent quark currents that emerge at the same leading terms of the determinant expansion are also presented. They might contribute to the vector and axial channels. Direct and simple momentum-dependent and -independent relations between different form factors are also presented. In particular, one relation corresponds to a generalized momentum-dependent Goldberger-Treiman relation (GTR). Besides that the corresponding strong quadratic radii of constituent quarks (scalar, pseudoscalar, vector, and axial) are also presented as functions of the quark effective mass. The axial (and vector) pion coupling presented in this work provides a further contribution for the corresponding axial (and vector) form factors and quadratic radii to those calculated in [28]. Two pion field definitions are considered, the Weinberg pion field, in terms of covariant derivatives, and the usual parametrization in terms of the operators $U=e^{i \vec{\pi} \cdot \vec{\sigma}}$. The conventional definition in terms of the functions $U=e^{i \vec{\pi} \cdot \vec{\tau}}$ provides the wellknown pseudoscalar pion coupling that is not found in the Weinberg pion field case. The isospin nondegeneracy of up-down quark masses is not considered in this work since it should be responsible for smaller (higher-order) effects. This work is organized as follows. In the next section, the steps of the method are briefly reminded and the large quark effective mass expansion of a sea quark determinant is performed. By keeping the full momentum dependence of the resulting constituent quark-pion couplings the corresponding form factors are presented for the two definitions of the pion field in the following section. Due to the momentum structure of some of the form factors it is also convenient to perform a truncation that provides, latter, corresponding positive averaged quadratic radii. All the eleven form factors, five for the Weinberg pion field and six for the second pion field definition, are written in terms of only two momentum-dependent functions, denoted $F_{1}(K, Q)$ and $F_{2}(K, Q)$. Besides that, the momentum-dependent constituent quark mass correction, $M_{3}(Q)$ is investigated. In the following section, numerical results are exhibited for different values of quark effective mass and for two very different gluon propagators: an effective longitudinal confining propagator considered by Cornwall [31] and a transversal one used extensively and successfully to provide hadron observables by Tandy and Maris [32]. Some ratios and comparisons of the form factors are also presented including the estimation of a momentum-dependent Goldberger-Treiman relation. The corresponding contributions for the pseudoscalar and axial strong constituent quark quadratic radii are also investigated as a functions of the quark effective mass for the different gluon propagators. In the last section, a summary is presented.

\section{THE QUARK DETERMINANT, PIONS AND CONSTITUENT QUARK CURRENTS}

Consider the nonperturbative one gluon exchange quark-quark interaction as one of the leading terms of QCD effective action whose generating functional is given by $[33,34]$

$$
\begin{aligned}
Z= & N \int \mathcal{D}[\bar{\psi}, \psi] \exp i \int_{x}[\bar{\psi}(i \not \partial-m) \psi \\
& \left.-\frac{g^{2}}{2} \int_{y} j_{\mu}^{b}(x) \tilde{R}_{b c}^{\mu \nu}(x-y) j_{\nu}^{c}(y)+\bar{\psi} J+J^{*} \psi\right],
\end{aligned}
$$

where $N$ is the normalization, $J, J^{*}$ the quark sources, $\int_{x}$ stands for $\int d^{4} x$, and $a, b \ldots=1, \ldots\left(N_{c}^{2}-1\right)$ stands for color in the adjoint representation being $N_{c}=3$. The functional measure for the quark field was written as $\mathcal{D}[\bar{\psi}, \psi]=\mathcal{D}[\bar{\psi}] \mathcal{D}[\psi]$. The quark gluon coupling constant is assumed to be $g$ and the development below is akin to the rainbow ladder Schwinger-Dyson equation (SDE). Below indices $i, j, k=0, \ldots\left(N_{f}^{2}-1\right)$ will be used for $\mathrm{SU}(2)$ isospin indices and, therefore, $N_{f}=2$. The quark current mass will be assumed to be equal for $\mathrm{u}, \mathrm{d}$ quarks. The color quark currents are given by $j_{a}^{\mu}=$ $\bar{\psi} \lambda_{a} \gamma^{\mu} \psi$, and the sums in color, flavor, and Dirac indices are implicit. A Landau-type gauge will be considered for a nonpertubative gluon propagator that can be 
written as $\tilde{R}_{a b}^{\mu \nu}(x-y) \equiv \tilde{R}_{a b}^{\mu \nu}=\delta_{a b}\left[\left(g^{\mu \nu}-\frac{\partial^{\mu} \partial^{\nu}}{\partial^{2}}\right) R_{T}(x-y)+\right.$ $\left.\frac{\partial^{\mu} \partial^{\nu}}{\partial^{2}} R_{L}(x-y)\right]$, where the transversal and longitudinal components are $R_{T}(x-y)$ and $R_{L}(x-y)$. This nonperturbative gluon kernel, therefore, incorporates to some extent the gluonic non-Abelian character with a corrected quark-gluon coupling such that they will provide enough strength to yield dynamical chiral symmetry breaking (DChSB). This has been found in several approaches and extensions [7,31,35-39].

The method was explained in detail in Refs. [25,26, $28,29,40]$, and therefore it will be succinctly described below. A Fierz transformation for the model (1) is performed and, by picking up the leading color singlet terms that provide the usual pion couplings, it allows to investigate the flavor structure in a more complete way. Besides that, color singlets, in one hand, avoid problems with unconfined spurious color d.o.f. and, on the other hand, provides a direct relation with quark-antiquark lightest observed states. These states are to be identified with the light hadrons d.o.f. and the scalar chiral condensate by means of the corresponding fields to be introduced. Chiral structures with combinations of bilocal currents are obtained. The quark field must be responsible for the formation of mesons and baryons and these different possibilities are envisaged by considering the background field method (BFM) [41,42]. Therefore, we consider the quark field is split into sea quark, $\psi_{2}$, composing (light) quark-antiquark states including light mesons and the chiral condensate, and the (constituent) background quark, $\psi_{1}$, to compose baryons. The shift of quark bilinears corresponds to performing a one-loop BFM calculation and it might be written for each of the color singlet Dirac/isospin channels $m=s, p, s i, p i, \mathrm{ps}, v, a, a s, v s \quad$ (scalar, pseudoscalar, scalar-isospin triplet, pseudoscalar-isospin triplet, vector, axial, vector-isospin triplet, axial-isospin triplet, where the isospin singlet states were omitted). Each of these channels might have a corresponding auxiliary field. However, only the lightest pseudoscalar-iso-triplet and isoscalar-scalar d.o.f. will be investigated in the present work. The quark field shift is of the following form:

$$
j^{m}=\bar{\psi} \Gamma^{m} \psi \rightarrow\left(\bar{\psi} \Gamma^{m} \psi\right)_{2}+\left(\bar{\psi} \Gamma^{m} \psi\right)_{1} .
$$

This separation preserves chiral symmetry. The sea quark can be integrated out exactly by means of the auxiliary field method that give rise to colorless quark-antiquark states, light mesons and the chiral quark condensate. Auxiliary fields are introduced by means of the unity integrals multiplying the generating functional. The only d.o.f. considered in this work are the chiral scalar and pseudoscalar- iso-triplet ones which are needed for the pion sector in the leading order. The heavier vector and axial mesons can be neglected in the lower energy regime. Therefore, one will be left with a model for pions and a scalar field interacting with constituent quarks. The corresponding unity integral for the scalar and pseudoscalar auxiliary bilocal fields $S(x, y), P_{i}(x, y)$ is the following,

$1=N^{\prime \prime} \int D[S] D\left[P_{i}\right] e^{-\frac{i}{2} \int_{x, y} R(x-y) \alpha\left[\left(S-g j_{(2)}^{S}\right)^{2}+\left(P_{i}-g j_{i,(2)}^{P}\right)^{2}\right]}$,

where $N^{\prime \prime}$ is a normalization, and

$$
R(x-y)=3 R_{T}(-y)+R_{L}(x-y) .
$$

Bilocal auxiliary fields for the different flavors can be expanded in an infinite orthogonal basis with all the excitations in the corresponding channel. For the pseudoscalar isotriplet fields, one has

$P_{i}(x, y)=P_{i}\left(\frac{x+y}{2}, x-y\right)=P_{i}(u, z)=\sum_{k} F_{k}(z) P_{i, k}(u)$,

where $F_{k}$ are vacuum functions invariant under translation for each of the local field $P_{i, k}^{\mu}(u)$. For the low energy regime one might pick up only the lowest energy modes, lightest $k=0$ which corresponds to the pions in this channel, i.e., $P_{i, k=0}=\pi_{i}$, making the form factors to reduce to constants in the zero momentum limit $F_{k}(z)=F_{k}(0)$. The saddle point equations for each of the remaining auxiliary fields, after the integration of the sea quark, can be written from the condition: $\frac{\partial S_{\text {eff }}}{\partial \phi_{q}}=0$. These equations for the NJL model and for the model (1) with Schwinger-Dyson equations at the rainbow ladder level have been analyzed in many works in the vacuum or under a finite energy density. The scalar field has the only saddle point equation with nontrivial solution for the quarkantiquark chiral condensate. This classical solution generates an effective mass for sea quarks.

Chiral symmetry leaves a freedom to define the pion field and chiral rotations can be done to modify its definition. The scalar field can be frozen by means of a chiral rotation and this produces the chiral condensate and a strongly nonlinear pion sector. An usual pion field definition is parametrized by the functions: $U=\exp (i \vec{\pi} \cdot \vec{\sigma})$ and $U^{\dagger}=\exp (-i \vec{\pi} \cdot \vec{\sigma})$. To investigate this aspect another pion field definition, the Weinberg ones, is characterized by writing all the chiral invariant sector in terms of a covariant pion derivative given by

$$
\mathcal{D}_{\mu} \pi_{i}=\frac{\partial_{\mu} \pi_{i}}{1+\vec{\pi}^{2}}
$$

The chiral symmetry breaking terms, however, can depend on combinations of $\vec{\pi}$ and $\vec{\pi}^{2}$. By doing the corresponding 
chiral rotations particular set of constituent quark-pion interactions are obtained. The corresponding Jacobian of the path integral measure will not be calculated and it might induce extra terms for the resulting form factors.

By performing a Gaussian integration of the sea quark field, the resulting determinant can be written, by means of the identity $\operatorname{det} A=\exp \operatorname{Tr} \ln (A)$ as

$$
\begin{gathered}
S_{\mathrm{eff}}=-i \operatorname{Tr} \ln \left\{-i S_{q}^{-1}(x-y)\right\} \\
S_{q}^{-1}(x-y) \equiv S_{0}^{-1}(x-y)+\Xi_{s}(x-y)+\sum_{q} a_{q} \Gamma_{q} j_{q}(x, y),
\end{gathered}
$$

where Tr stands for traces of all discrete internal indices and integration of spacetime coordinates and $\Xi_{s}(x-y)$ stands for the coupling of sea quark to the scalar-pseudoscalar fields for a particular pion field. This coupling term can be written, respectively, for the Weinberg pion field $\left(\Xi_{s}^{W}(x-y)\right)$ and for the usual pion field $\left(\Xi_{s}^{U}(x-y)\right)$ in terms of unitary functions $U, U^{\dagger}$ as $[25,26]$

$$
\begin{aligned}
\Xi_{s}^{W}(x-y)= & {\left[\gamma^{\mu} \vec{\sigma} \cdot \mathcal{D}_{\mu} \vec{\pi} i \gamma_{5}+i \gamma^{\mu} \vec{\sigma} \cdot \frac{\vec{\pi} \times \partial_{\mu} \vec{\pi}}{1+\vec{\pi}^{2}}\right.} \\
& \left.+4 m\left(\frac{\vec{\pi}^{2}}{1+\vec{\pi}^{2}}-\frac{\epsilon_{i j k} \sigma_{k} \pi_{i} \pi_{j}}{1+\vec{\pi}^{2}}\right)\right] \delta(x-y), \\
\Xi_{S}^{U}(x-y)= & F\left(P_{R} U+P_{L} U^{\dagger}\right) \delta(x-y),
\end{aligned}
$$

where $F=f_{\pi}$ is the pion field normalization, and $P_{R / L}=$ $\left(1 \pm \gamma_{5}\right) / 2$ are the chirality right- and left-hand projectors.

The free quark kernel can be written as $S_{0}^{-1}(x-y)=$ $(i \not \supset-m) \delta(x-y)$, where $m$ is so far the current quark mass. The classical solution for the scalar field, found from its gap equation, is directly incorporated into an effective quark mass $M^{*}=m-\langle s\rangle$. The redefined quark kernel can be written as

$$
S_{0}^{-1}(x-y)=\left(i \not \partial-M^{*}\right) \delta(x-y) .
$$

In expression (8), the following quantity, with the usual chiral constituent quark currents that yield the leading couplings to pions, has been used:

$$
\begin{aligned}
& \frac{\sum_{q} a_{q} \Gamma_{q} j_{q}(x, y)}{\alpha g^{2}} \\
& =2 R(x-y)\left[\bar{\psi}(y) \psi(x)+i \gamma_{5} \sigma_{i} \bar{\psi}(y) i \gamma_{5} \sigma_{i} \psi(x)\right] \\
& \quad-\bar{R}^{\mu \nu}(x-y) \gamma_{\mu} \sigma_{i}\left[\bar{\psi}(y) \gamma_{\nu} \sigma_{i} \psi(x)+\gamma_{5} \bar{\psi}(y) \gamma_{5} \gamma_{\nu} \sigma_{i} \psi(x)\right] .
\end{aligned}
$$

In this expression, $\alpha=2 / 9$ from the Fierz transformation, $R(x-y)$ was given in (4) and

$$
\begin{aligned}
\bar{R}^{\mu \nu}(x-y)= & g^{\mu \nu}\left(R_{T}(x-y)+R_{L}(x-y)\right) \\
& +2 \frac{\partial^{\mu} \partial^{\nu}}{\partial^{2}}\left(R_{T}(x-y)-R_{L}(x-y)\right) .
\end{aligned}
$$

\section{LEADING FORM FACTORS}

In the following, consider the quark (and gluon) large effective mass expansion for the case in which quark and pion fields exchange momenta. To provide the reader with one example, one of the leading pion constituent quark effective interactions is the pseudoscalar coupling and it shows up in the first-order terms of the expansion as follows,

$$
\begin{aligned}
I_{\mathrm{det}}^{\mathrm{ps}}= & \frac{i}{2} \operatorname{Tr}\left[S_{0}(y-x) i \gamma_{5} \sigma_{i} i \gamma_{5} \sigma_{i} \pi_{i}(x) S_{0}(x-z)\right. \\
& \left.\times R(y-z) i \gamma_{5} \sigma_{j} \bar{\psi}(z) i \gamma_{5} \sigma_{j} \psi(y)\right],
\end{aligned}
$$

with the insertion of complete sets of orthogonal momentum states, a pseudoscalar form factor at the constituent quark level emerges in momentum space, $G_{\mathrm{ps}}^{U}(K, Q)$, where the momenta $K, Q$ are defined below. For this, the trace in internal indices (isospin, color and Dirac) were calculated. By considering incoming quark with momentum $K$, and pion(s) with total momenta $Q$ the set of leading momentumdependent effective couplings for the first pion definition (W) in the weak pion field limit $\left(1+\vec{\pi}^{2} \simeq 1\right)$ is given by

$$
\begin{aligned}
\mathcal{L}_{W}^{q-\pi}= & M_{3}(K) \bar{\psi}(K) \psi(K)+2 i \epsilon_{i j k} G_{V}^{W}(K, Q) \pi_{i}\left(q_{a}\right) \partial^{\nu} \pi_{j}\left(q_{b}\right) \bar{\psi}(K) \gamma_{\nu} \sigma^{k} \psi(K+Q) \\
& +2 G_{A}^{W}(K, Q) \partial^{\nu} \pi^{i}(Q) \bar{\psi}(K) i \gamma_{5} \gamma_{\nu} \sigma^{i} \psi(K+Q)+F G_{\beta s b F}(K, Q) \pi_{i}\left(q_{1 a}\right) \pi_{i}\left(q_{b}\right) \bar{\psi}(K) \psi(K+Q) \\
& -G_{\mathrm{ps}}^{p, W}(K, Q) \frac{\partial_{\mu} \partial^{\mu} \pi^{i}(Q)}{M^{*}} \bar{\psi}(K) i \gamma_{5} \sigma^{i} \psi(K+Q)-G_{s}^{p, W}(K, Q) \frac{\partial_{\mu} \partial^{\mu} \pi^{2}(Q)}{M^{*}} \bar{\psi}(K) \psi(K+Q),
\end{aligned}
$$

where $Q=Q_{\pi}$ is the total momentum carried by one or two pion in each of the vertices, and it will be for both pion field definitions $W$ and $U$, being that, in the vector and scalar constituent quark currents couplings, $Q=q_{a}+q_{b}$ and the pion field was kept dimensionless. The last two terms, momentum-dependent ones, were obtained with an integration by parts. In this expression, $M_{3}(K)$ is a running effective mass that will be defined below in (23), and the 
following dimensionless form factors were defined in terms of the functions $F_{1}(K, Q)$ given below,

$$
\begin{aligned}
& G_{A}^{W}(K, Q)=G_{V}^{W}(K, Q)=4 d_{1} N_{c}\left(\alpha g^{2}\right) F_{1}(K, Q) \\
& G_{\beta s b F}(K, Q)=64 d_{1} N_{c} \frac{m}{F}\left(\alpha g^{2}\right) F_{1}(K, Q), \\
& G_{\mathrm{ps}}^{p, W}(K, Q)=\frac{M^{*}}{4 m} G_{s}^{p, W}(K, Q) \\
&=16 d_{1} M^{*} N_{c}\left(\alpha g^{2}\right) F_{2}(K, Q),
\end{aligned}
$$

where $N_{c}=3, d_{n}=(-1)^{n+1} /(2 n)$. It is interesting to note that the scalar pion coupling is proportional to the current quark mass and, therefore it is a consequence of explicit chiral symmetry breaking. There are scalar and a pseudoscalar momentum-dependent form factors. Although the usual pseudoscalar pion coupling to pseudoscalar quark current does not emerge at this level of calculation for the $\mathrm{W}$ pion field definition, there is the coupling $G_{\mathrm{ps}}^{p, W}(K, Q)$ that might contribute for the axial channel. Because it is simply proportional to other form factors by means of the function $F_{2}(K, Q)$ it will not be investigated explicitly numerically below. An analogous conclusion can be drawn for the derivative-scalar term $G_{s}^{p, W}(K, Q)$ that might contribute for the vector channel.

The complete set of leading momentum dependent couplings with their form factors for the second pion definition, with the same convention for momenta of expression (15) and dimensionless pion filed, is given by

$$
\begin{aligned}
\mathcal{L}_{U}^{q-\pi}= & M_{3}(K) \bar{\psi}(K) \psi(K)+G_{2 j s}(K, Q) F \pi_{i}\left(q_{a}\right) \pi_{i}\left(q_{b}\right) \bar{\psi}(K) \psi(K+Q)+G_{\mathrm{ps}}^{U}(K, Q) F \pi_{i}(Q) \bar{\psi}(K) \sigma_{i} i \gamma_{5} \psi(K+Q) \\
& +i \epsilon_{i j k} 2 G_{V}^{U}(K, Q) \pi_{i}\left(q_{a}\right)\left(\partial_{\mu} \pi_{j}\left(q_{b}\right)\right) \bar{\psi}(K) \gamma_{\mu} \sigma^{j} \psi(K+Q), \\
& +2 G_{A}^{U}(K, Q)\left(\partial^{\mu} \pi_{i}(Q)\right) \bar{\psi}(K) i \gamma_{5} \gamma_{\nu} \sigma^{i} \psi(K+Q) \\
& -G_{\mathrm{ps}}^{p}(K, Q) \frac{\left(\partial^{2} \pi_{i}(Q)\right)}{M^{*}} \bar{\psi}(K) \sigma_{i} i \gamma_{5} \psi(K+Q)-G_{s}^{p}(K, Q) \frac{\partial^{2}\left(\pi_{i}\left(q_{a}\right) \pi_{i}\left(q_{b}\right)\right)}{M^{*}} \bar{\psi}(K) \psi(K+Q),
\end{aligned}
$$

where $M_{3}(K)$ is the same as the mass in expression (15) and it will be defined in expression (23). The other form factors were defined as

$G_{\mathrm{ps}}^{U}(K, Q)=G_{2 j s}(K, Q)=32 d_{1} N_{c}\left(\alpha g^{2}\right) F_{1}(K, Q)$,

$G_{A}^{U}(K, Q)=G_{V}^{U}(K, Q)=16 d_{1} N_{c} F\left(\alpha g^{2}\right) F_{2}(K, Q)$

$G_{\mathrm{ps}}^{p}(K, Q)=G_{s}^{p}(K, Q)=16 d_{1} N_{c} F\left(\alpha g^{2}\right) F_{2}(K, Q)$.

The derivative couplings with form factors $G_{\mathrm{ps}}^{p}(K, Q)$ and $G_{s}^{p}(K, Q)$ have simply a different normalization with respect to the ones from the $\mathrm{W}$ pion field definition: $G_{\mathrm{ps}}^{p, W}(K, Q)$ and $G_{s}^{p, W}(K, Q)$. For example, it can be seen that $G_{\mathrm{ps}}^{p, W}(K, Q)=\frac{M^{*}}{F} G_{\mathrm{ps}}^{p}(K, Q)$. At this level, it is interesting to note that $G_{\mathrm{ps}}(K, Q)=G_{2 j s}(K, Q)$ in reasonable agreement with other results [43], and also $G_{A}(K, Q)=$ $G_{V}(K, Q)$ for both pion field definitions.

The loop momentum integrals of each of the form factors above will be written and investigated for constituent quark with $K=0$, except for the effective mass $M_{3}(Q)$. After a Wick rotation for the Euclidean momentum space, these functions are given by

$$
\begin{aligned}
F_{1}(0, Q) & =\int_{k}\left(k \cdot(k+Q)-M^{* 2}\right) \tilde{S}_{0}(k) \tilde{S}_{0}(k+Q) \overline{\bar{R}}(-k), \\
F_{2}(0, Q) & =\int_{k} M^{*} \tilde{S}_{0}(k) \tilde{S}_{0}(k+Q) \overline{\bar{R}}(-k) \\
M_{3}(Q) & =16 d_{1} N_{c} M^{*}\left(\alpha g^{2}\right) \int_{k} \tilde{S}_{0}(k+Q) R(-k),
\end{aligned}
$$

where $\int_{k}=\int \frac{d^{4} k}{(2 \pi)^{4}}$ and the following functions in momentum space for components of the quark and gluon propagator used:

$$
\begin{gathered}
\tilde{S}_{0}(k)=\frac{1}{k^{2}+M^{* 2}}, \\
\overline{\bar{R}}(k)=2 R(k)=6 R_{T}(k)+2 R_{L}(k) .
\end{gathered}
$$

The only form factor that might have an ultraviolet divergence UV is $M_{3}(Q)$ if the gluon propagator does not possess particular UV behavior. The other are completely finite if the nonperturbative gluon propagator is infrared regular.

The momentum structure of the form factor $F_{1}(0, Q)$ has a positive first derivative with respect to $Q^{2}$ for very small $Q$, and therefore it yields negative quadratic radii. To overcome that, $F_{1}(0, Q)$ might be truncated by approximating the quark kernel by $S_{0}(k) \simeq M^{*} \tilde{S}_{0}(k)$. It yields for the function $F_{1}(0, Q)$ the following expression: 

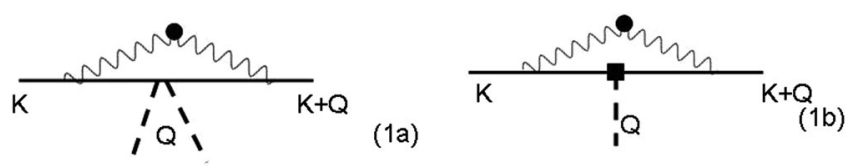

(1c)
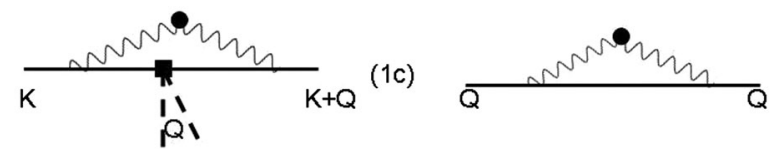

$(1 d$

FIG. 1. These diagrams correspond to the quark-pion effective couplings from expression (15). The wavy line with a full dot is a (dressed) nonperturbative gluon propagator, the solid lines stand for a constituent quark (external line) or sea quark (internal line), and dashed lines represents pion field, the full square in a vertex represents a derivative coupling. Diagram 1(d) represents the effective quark mass correction.

$$
F_{1}^{\mathrm{tr}}(0, Q)=M^{* 2} \int_{k} \tilde{S}_{0}(k) \tilde{S}_{0}(k+Q) \overline{\bar{R}}(-k) .
$$

This truncation might be expected to correspond to making an effective mass $M^{*}$ to be momentum dependent in the expression of $F_{1}(K, Q)$.

In Fig. 1, the diagrams corresponding to the expressions (15) for the Weinberg pion field definition are presented, where the pion-quark vertices with a square are the derivative ones and diagram $1(\mathrm{~d})$ stands for the effective mass $M_{3}(Q)$. The dressed (nonperturbative) gluon propagator is indicated by a wavy line with a full circle and pion is represented by dashed lines. In diagrams $1(\mathrm{a})-1(\mathrm{c})$, the incoming constituent has momentum $K$ and the outgoing constituent quark has momentum $K+Q$, being $Q$ the total momentum transferred by pion(s). Figure 2 exhibits the diagrams for the pion constituent quark couplings for the usual pion field definition given in expression (19) with the same conventions as Fig. 1.

\section{NUMERICAL RESULTS}

To provide numerical results, two gluon propagators were chosen. A transversal one from Tandy-Maris $D_{I}(k)$ [32] and the other is an effective longitudinal confining one by Cornwall $D_{I I}(k)$ [31]. Both of them yield DChSB and they are written below with the following association:

$$
g^{2} \tilde{R}^{\mu \nu}(k) \equiv h_{a} D_{a}^{\mu \nu}(k)
$$

where $D_{a}^{\mu \nu}(k)(a=I, I I)$ is one of the chosen gluon propagators from the quoted articles, $h_{a}$ is a real positive constant factor used in previous works [26,29] to fix the quark gluon (running) coupling constant such as to reproduce one expected value either of the vector/axial pion coupling constant in the vacuum or vector meson coupling to constituent quarks constant, $g_{V} h_{a}=1, g_{A} h_{a}=1$ or $g_{\rho} h_{a} \simeq 12$. In the present work, this factor was chosen

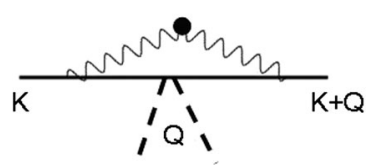

(2a)
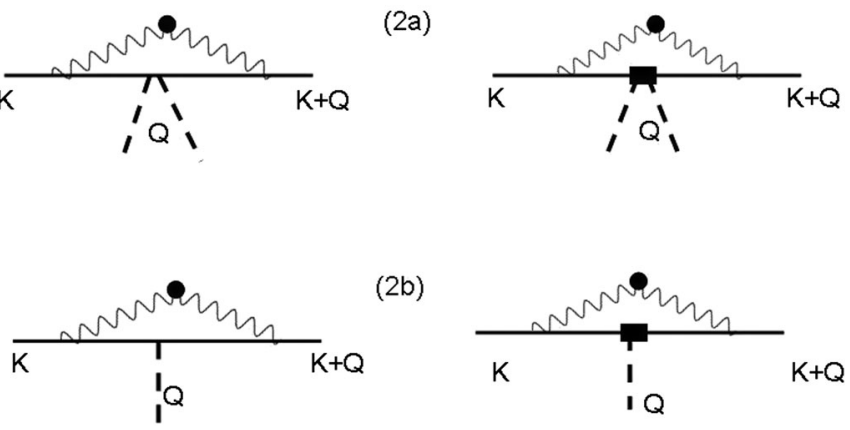

(2b)
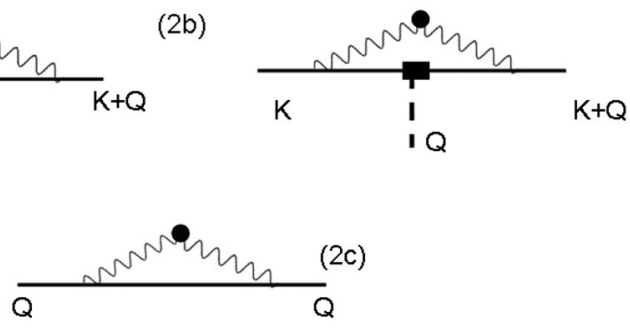

FIG. 2. These diagrams correspond to the quark-pion effective couplings from expression (19). The wavy line with a full dot is a (dressed) nonperturbative gluon propagator, the solid lines stand for a constituent quark (external line) or sea quark (internal line), and dashed lines represents pion field, the full square in a vertex represents a derivative coupling.

for each of the gluon propagators and pion field definition to provide $g_{A}(0) h_{a}=1$. Their values will be shown in the caption of the corresponding figure.

The expressions for the two gluon propagators are the following:

$$
\begin{gathered}
D_{I}(k)=\frac{8 \pi^{2}}{\omega^{4}} D e^{-k^{2} / \omega^{2}}+\frac{8 \pi^{2} \gamma_{m} E\left(k^{2}\right)}{\ln \left[\tau+\left(1+k^{2} / \Lambda_{\mathrm{QCD}}^{2}\right)^{2}\right]}, \\
D_{I I}(k)=\frac{K_{F}}{\left(k^{2}+M_{k}^{2}\right)^{2}},
\end{gathered}
$$

where for the first expression $\gamma_{m}=12 /\left(33-2 N_{f}\right), N_{f}=4$, $\Lambda_{\mathrm{QCD}}=0.234 \mathrm{GeV}, \tau=e^{2}-1, E\left(k^{2}\right)=\left[1-\exp \left(-k^{2} /\right.\right.$ $\left.\left[4 m_{t}^{2}\right]\right) / k^{2}, m_{t}=0.5 \mathrm{GeV}, \omega=0.5 \mathrm{GeV}, \quad D=0.55^{3} / \omega$ $\left(\mathrm{GeV}^{2}\right)$; and for the second expression $K_{F}=$ $\left(2 \pi M_{k} /\left(3 k_{e}\right)\right)^{2}$ where $k_{e}=0.15$ and $M_{k}=220 \mathrm{MeV}$.

In Fig. 3, the resulting constituent quark (running) effective mass $M_{3}^{*}(Q)$ is shown as a function of the constituent quark momentum for an UV cutoff $\Lambda=2 \mathrm{GeV}$, in dashed and continuous lines and it is compared to a result from Schwinger-Dyson equations at the rainbow ladder approximation from Ref. [44]. The multiplicative factors $1 / 4$ and $3 / 4$ were chosen to fit the curves into a suitable scale and they are needed because of the large value of $\Lambda$.

In Fig. 4, the axial form factor contribution for zero quark momentum $G_{A}^{W}(0, Q)$ and its truncated version $G_{A}^{W, t r}(0, Q)$ for the Weinberg pion field are presented for different values of the quark effective mass from the gap equation $M^{*}$ and for the gluon propagator $D_{I I}(k)$. In all cases of the figures with the axial form factor, the linear dependence on the pion momentum from the coupling was 


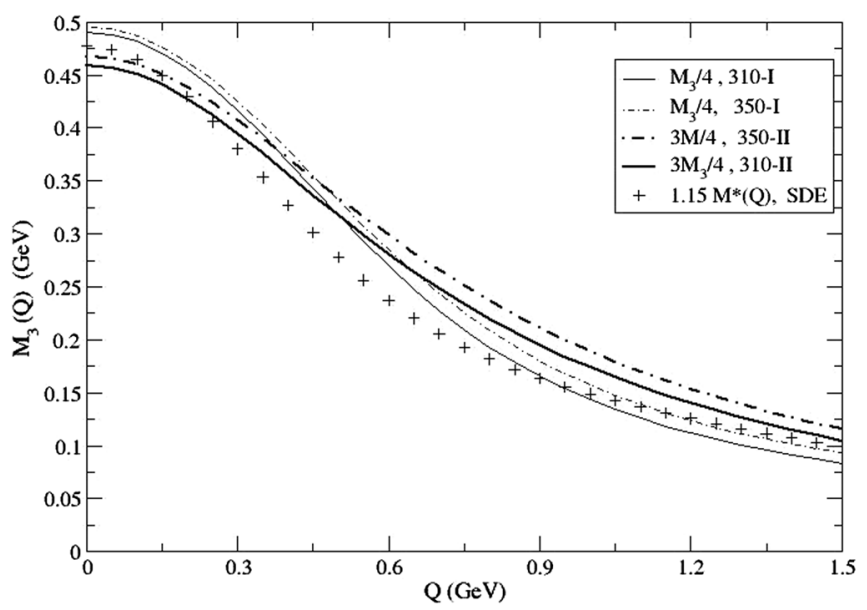

FIG. 3. The dynamical running constituent quark effective mass $M_{3}(Q)$ divided by $4, M_{3}(Q) / 4$, for the gluon propagator $I$, in continuous and dashed thin lines and multiplied by $3 / 4, \frac{3}{4} M_{3}(Q)$, in thick lines for the gluon propagator $I I$, in dashed $\left(M^{*}=\right.$ $350 \mathrm{MeV})$ and continuous $\left(M^{*}=310 \mathrm{MeV}\right)$ lines. A gap effective mass $M^{*}(Q)$ from SDE from Ref. [44] multiplied by a factor 1.15 to allow for a better comparison of the momentum dependence.

not included. In Fig. 5, the same results are exhibited for the gluon propagator $D_{I}(k)$. Figures 4 and 5 present the same behavior without meaningful differences except for the relative normalization of the nontruncated form factor. Besides that, a dipolar fitting for experimental results of axial pion-nucleon coupling is drawn with symbols + with a normalization to allow for comparison of the momentum dependence. It is given by $[16,18,20]$

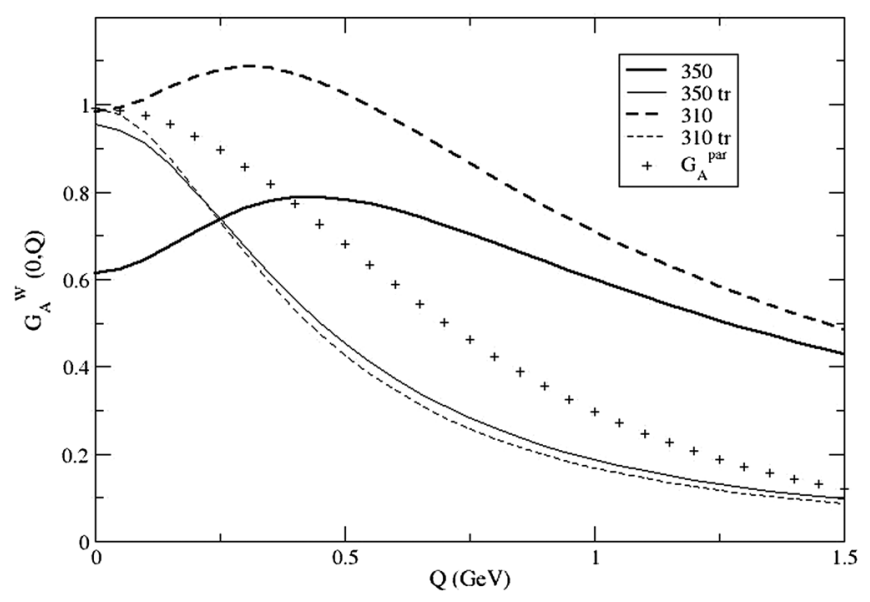

FIG. 4. The normalized axial form factor $G_{A}^{W}(0, Q)$ for the Weinberg pion field as a function of the pion momentum is presented in this figure for the gluon propagator $D_{I I}(k)$, untruncated expression with $h_{a}=\frac{1}{0.2}$ and truncated one with $h_{a}=\frac{1}{0.46}$, and for different values of the sea quark effective mass $M^{*}$ from the gap equation. Solid thin and thick lines for $M^{*}=350 \mathrm{MeV}$; dashed lines for $310 \mathrm{MeV}$. Signs + for the normalized fitting of expression $(30), G_{A}^{\mathrm{par}}\left(Q^{2}\right)$.

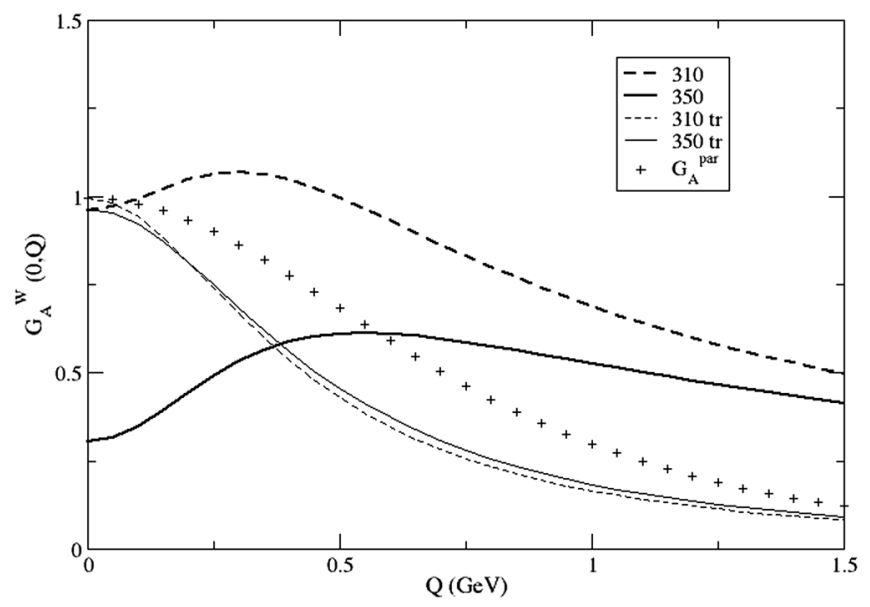

FIG. 5. The axial form factor $G_{A}^{W}(0, Q)$ for the Weinberg pion field as a function of the pion momentum is presented in this figure for the gluon propagator $D_{I}(k)$ from Ref. [32], being that for the untruncated expression it is taken $h_{a}=\frac{1}{0.38}$ and for the truncated one $h_{a}=\frac{1}{1.4}$, and for different values of the sea quark effective mass $M^{*}$ from the gap equation. Solid thin and thick lines for $M^{*}=350 \mathrm{MeV}$; dashed lines for $310 \mathrm{MeV}$. Signs + for the fitting of expression (30), $G_{A}^{\mathrm{par}}\left(Q^{2}\right)$.

$$
G_{A}^{\mathrm{par}}\left(Q^{2}\right)=\frac{G_{0}}{\left(1+\frac{Q^{2}}{M_{A}^{2}}\right)^{2}},
$$

by considering $M_{A}=1.1 \mathrm{GeV}$ and by adopting a normalization for $G_{A}^{\mathrm{par}}\left(Q^{2}=0\right)$ obtained in the present work for each of the gluon propagators, for the case of $M^{*}=$ $0.31 \mathrm{GeV}$. The fitting for experimental values decreases slower than the (constituent quark) form factors $G_{A}^{W}(0, Q)$ and two reasons might directly identified for that. It might signal there is missing strength from more complete quark and gluon kernels. However, it also might indicate the need to account other effects rather related to nucleon structure d.o.f. These two possibilities are not exclusive of one another; however, they correspond to different types of constituent quark models for hadrons (baryons) since they correspond to different roles of constituent quark interactions for the baryon structure. In any case, apart from a possible difference on the overall normalization, the difference is not very large and it appears in intermediary momenta. It can be noted that the nontruncated expressions provide a positive momentum slope at $Q=0$, these expressions therefore would provide a negative averaged quadratic axial radii. The truncated expressions correct this behavior.

In Fig. 6, the axial form factor correction for the second pion field definition, $G_{A}^{U}(0, Q)$, as a function of pion momentum is shown for gluon propagators $D_{I I}(k)$ and $D_{I}(k)$ for different values of the quark effective mass $M^{*}$. The same fitting $G_{A}^{\mathrm{par}}(Q)$ is plotted (+) with the value at $Q=0$ adjusted from the $G_{A}^{U}(0, Q=0)$ to make an appropriated comparison. The truncated version of 


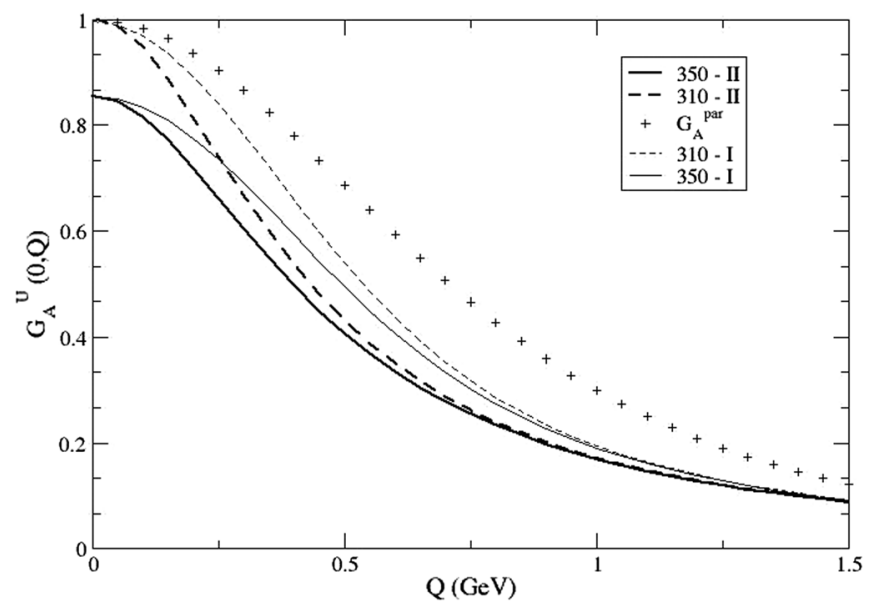

FIG. 6. The axial (equal to the vector) form factor $G_{A}^{U}(0, Q)$ (usual pion field) as a function of the pion momentum is presented in this figure for the gluon propagators $D_{I}(k)$, with factor $h_{a}=\frac{1}{0.83}$, and $D_{I I}(k)$, with $h_{a}=\frac{1}{0.27}$, and for different values of the sea quark effective mass $M^{*}$, from the gap equation. Solid line is used for $M^{*}=350 \mathrm{MeV}$, dashed line for $M^{*}=$ $310 \mathrm{MeV}$. Sign + for the fitting of expression (30), $G_{A}^{\mathrm{par}}\left(Q^{2}\right)$.

$G_{A}^{W, \text { tr }}(0, Q)$ from Figs. 4 and 5 has a similar behavior to $G_{A}^{U}(0, Q)$, and in fact they both are written in terms of $F_{2}(0, Q)$ with different normalizations. Although the overall behavior is similar to the experimental fit, for both $G_{A}^{W, \text { tr }}(0, Q)$ and $G_{A}^{U}(0, Q)$, the form factor contribution $G_{A}^{U}(0, Q)$ has a behavior slightly closer to the experimental fit.

The axial coupling constant at the constituent quark level has been argued to be close to $g_{A} \simeq 3 / 4$ [12] or $g_{A} \simeq 1$ [21]. Results from the form factors fall well within the correct order of magnitude and value. Also, in the present work, it was shown in expressions (16) and (21) that the axial and vector form factors are equal to each other, due to chiral symmetry, for each the two pion field definitions considered.

\section{A. Pseudoscalar coupling}

In Figs. 7 and 8, the pseudoscalar form factor $G_{\mathrm{ps}}^{U}(0, Q)$ and its truncated version $G_{\mathrm{ps}}^{U, \text { tr }}(0, Q)$ are presented for the gluon propagators $D_{I I}(k)$ and $D_{I}(k)$, respectively. The zero momentum $Q=0$ values are basically 1 order of magnitude larger than the zero momentum axial form factor as expected from phenomenology. Results with $D_{I}(k)$ have considerably larger absolute values than with $D_{I I}(k)$. The dipolar fitting for data from lattice QCD calculations (30) [45] is also shown with a suitable normalization at $G_{\mathrm{ps}}(0,0)$ to compare with the results from expressions above for the case $M^{*}=0.31 \mathrm{GeV}$. All the results from the truncated expressions for $G_{\mathrm{ps}}^{U}(0, Q)$ yield similar results for $M^{*}=$ 0.31 and $0.35 \mathrm{GeV}$. Whereas the truncated version presents a monotonic decrease with momentum $Q$ the complete

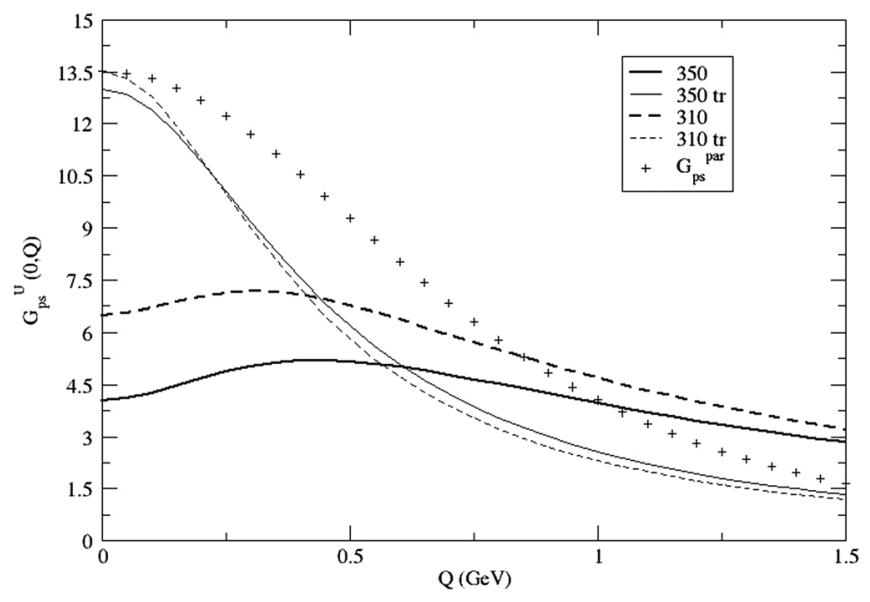

FIG. 7. The pseudoscalar form factor $G_{\mathrm{ps}}^{U}(0, Q)$ as a function of the quark momentum is presented in this figure for the gluon propagator $D_{I I}(k)$, with factor $h_{a}=\frac{1}{0.27}$, and for different values of the sea quark effective mass $M^{*}$, from the gap equation. Results from both the complete and the truncated (tr) expressions are shown. Solid lines are used for $M^{*}=350 \mathrm{MeV}$, dashed lines for $M^{*}=310 \mathrm{MeV}$. Signs + for the corresponding fitting of expression (30), $G_{A}^{\mathrm{par}}\left(Q^{2}\right)$.

expression has an increase up to around $Q \sim 0.40-0.45 \mathrm{GeV}$ and then it decreases for larger $Q$. It has, therefore, the same behavior of $G_{A}^{W}(Q)$ shown in the previous section. The deviation of the form factor $G_{\mathrm{ps}}^{U, \mathrm{tr}}(0, Q)$ momentum dependence from the fitting $G_{\mathrm{ps}}^{\mathrm{par}}(0, Q)$ is slightly larger than the deviation of the axial $G_{A}^{U}(0, Q)$ form factor with respect to the corresponding nucleon-pion experimental fitting. The reasons must be the same, the momentum

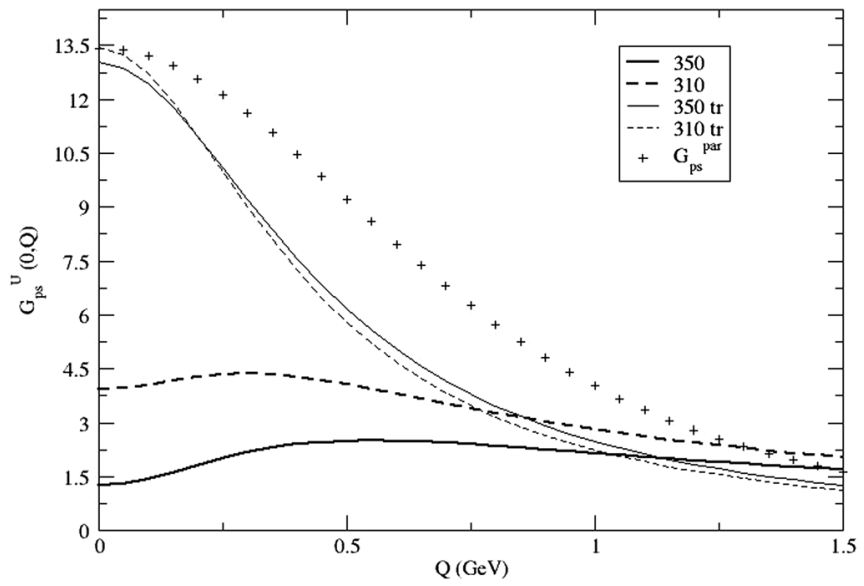

FIG. 8. The pseudoscalar form factor $G_{\mathrm{ps}}^{U}(0, Q)$ as a function of the quark momentum is presented in this figure for the gluon propagator $D_{I}(k)$, with factor $h_{a}=\frac{1}{0.83}$, and for different values of the sea quark effective mass $M^{*}$, from the gap equation. Results from both the complete and the truncated (tr) expressions are shown. Solid lines are used for $M^{*}=350 \mathrm{MeV}$, dashed lines for $M^{*}=310 \mathrm{MeV}$. Signs + for the corresponding fitting of expression (30), $G_{A}^{\mathrm{par}}\left(Q^{2}\right)$. 
dependence of the quark and gluon kernels and/or internal nucleon effects.

Standard hadron effective coupling constants are usually obtained for particular values of the transferred momentum such as $Q^{2}=0$ or $Q^{2} \simeq-m_{\pi}^{2}$. The only numerical values for the form factors at the spacelike momenta $Q^{2}<0$ shown in this work are these next ones for the usual pseudoscalar pion coupling at $Q^{2}=-m_{\pi}^{2}$, i.e., closer to the physical definition of $G_{\pi N}$ that is taken from spacelike momenta at the muon or pion mass. For the quark effective mass $M^{*}=0.31 \mathrm{GeV}$ and the two gluon propagators two values were obtained: for the complete expression (20) and for the momentum truncated expression $G_{\mathrm{ps}}^{W, \text { tr }}(0, Q)$ with (26). By considering the same factors $h_{a}$ adopted for the figures of the pseudoscalar form factors $\left(h_{I}=1 / 0.83\right.$ and $\left.h_{I I}=1 / 0.27\right)$, they are given by

$$
\begin{gathered}
\text { I } \quad G_{\mathrm{ps}}^{U}\left(0, Q^{2}=-m_{\pi}^{2}\right)=1.9, \quad G_{\mathrm{ps}}^{U}(0,0)=3.4, \\
\text { I } \quad G_{\mathrm{ps}}^{U \text {,tr }}\left(0, Q^{2}=-m_{\pi}^{2}\right)=16.4, \quad G_{\mathrm{ps}}^{U, \mathrm{tr}}(0,0)=13.4,
\end{gathered}
$$

$$
\begin{aligned}
& \text { II } \quad G_{\mathrm{ps}}^{U}\left(0, Q^{2}=-m_{\pi}^{2}\right)=4.1, \quad G_{\mathrm{ps}}^{U}(0,0)=5.9, \\
& \text { II } \quad G_{\mathrm{ps}}^{U, \mathrm{tr}}\left(0, Q^{2}=-m_{\pi}^{2}\right)=15.2, \quad G_{\mathrm{ps}}^{\mathrm{tr}}(0,0)=13.3 .
\end{aligned}
$$

The difference between the form factor $G_{\mathrm{ps}}^{U}(0, Q)$ and its truncated version, $G_{\mathrm{ps}}^{U, \mathrm{tr}}(0, Q)$, is of course present in this spacelike values. The values from the truncated expression are also closer to experimental data for the nucleon-pion coupling constant and results from other calculations.

\section{B. Goldberger-Treiman and other relations in timelike momenta}

Next ratios of the form factors are calculated. The following momentum-dependent ratios between dimensionless quantities were considered:

$$
\begin{gathered}
G T_{W}(Q) \equiv \frac{G_{V}^{W}(0, Q)}{G_{\beta s b F}(0, Q)}=\frac{F}{16 m} \simeq 1, \\
G T(Q)=\frac{M^{*}}{F}\left(\frac{G_{A}^{U}(0, Q)}{G_{\mathrm{ps}}^{U}(0, Q)}\right)=\frac{M^{*}}{F} \frac{G_{V}(0, Q)}{G_{2 j s}(0, Q)} \\
=\frac{M^{*}}{F} \frac{F}{2} \frac{F_{2}(0, Q)}{F_{1}(0, Q)}, \\
\frac{G_{A}^{U}(0, Q)}{G_{V}^{U}(0, Q)}=\frac{G_{\mathrm{ps}}^{U}(0, Q)}{G_{2 j s}(0, Q)}=1,
\end{gathered}
$$

where the first one $G T_{W}(Q)$ is an equivalent of the GTR expression for the Weinberg pion field in which the pseudoscalar pion coupling does not appear but the (symmetry breaking) scalar two pion coupling to constituent quark appears. This ratio is momentum independent, and it depends on the current quark mass $m \sim 5.75 \mathrm{MeV}$ for which $16 m \simeq f_{\pi}=F=92 \mathrm{MeV}$ and, therefore, $G T_{W} \simeq 1$. The function $G T(Q)$ for the second pion definition has a constant factor $F / M^{*}$ such that if the GTR relation is satisfied the ratio $G T(Q) \rightarrow 1$ and this is verified for very large $M^{*}$. The last expression has two chiral symmetry relations for form factors, and their corresponding effective coupling constants for the second pion field definition.

In Fig. 9, the ratio $G T(Q)$ is presented as a function of momentum for different effective quark masses $M^{*}$. The ratio $G T(Q)$ does not satisfy necessarily the GTR at $Q=0$ because the quark effective masses are not large enough. This ratio $G T(Q)$ has the same behavior found in other works [15]. The deviation from the GTR intrinsically due to the momentum dependence of each of the form factors for the nucleon level Goldberger-Treiman relation is usually denoted by $R(Q)$. It is usually parametrized in terms of the nucleon mass $M$ [15], and by substituting $M$ by the quark effective mass $M^{*}$ it is given by the following expression:

$$
G_{A}\left(Q^{2}\right)=\frac{f_{\pi}}{M^{*}} G_{\pi N N}\left(Q^{2}\right)-\frac{Q^{2}}{4 M^{*}} R\left(Q^{2}\right)
$$

where $G_{\pi N}\left(Q^{2}\right)$ is to be substituted by $G_{\mathrm{ps}}(Q)$. By considering the constituent quark mass $M^{*}=0.28 \mathrm{GeV}$ and $0.31 \mathrm{GeV}$ this function is exhibited in Fig. 10 for the second pion definition. It goes to zero quite fast with increasing $(Q)$ depending not only on the quark effective mass $M^{*}$ but also on the gluon propagator considered.

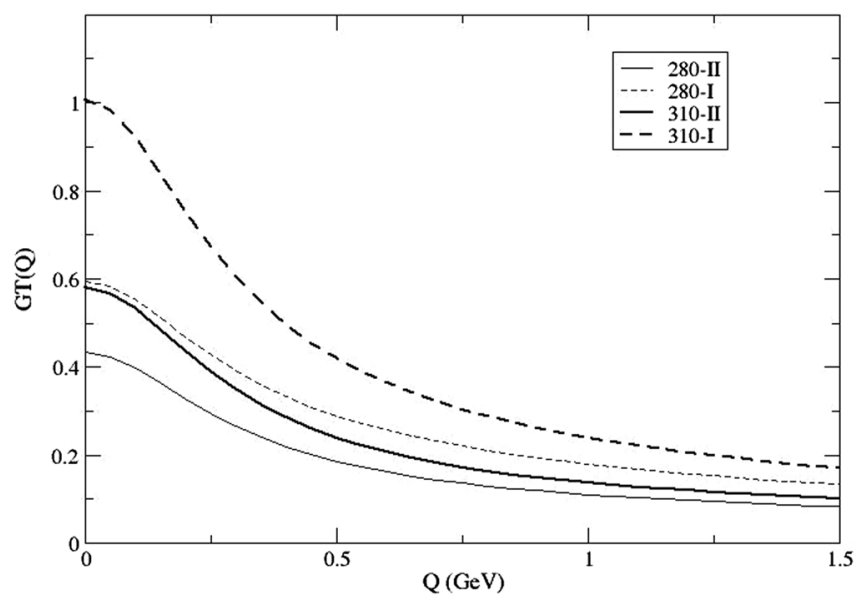

FIG. 9. The ratio $G T(0, Q)$ is shown as a function of momenta for two different quark effective masses $M^{*}=280,310 \mathrm{MeV}$ and for the two gluon propagators. The limit in which the GoldbergerTreiman relation is recovered corresponds to $G T(0)=1$. 


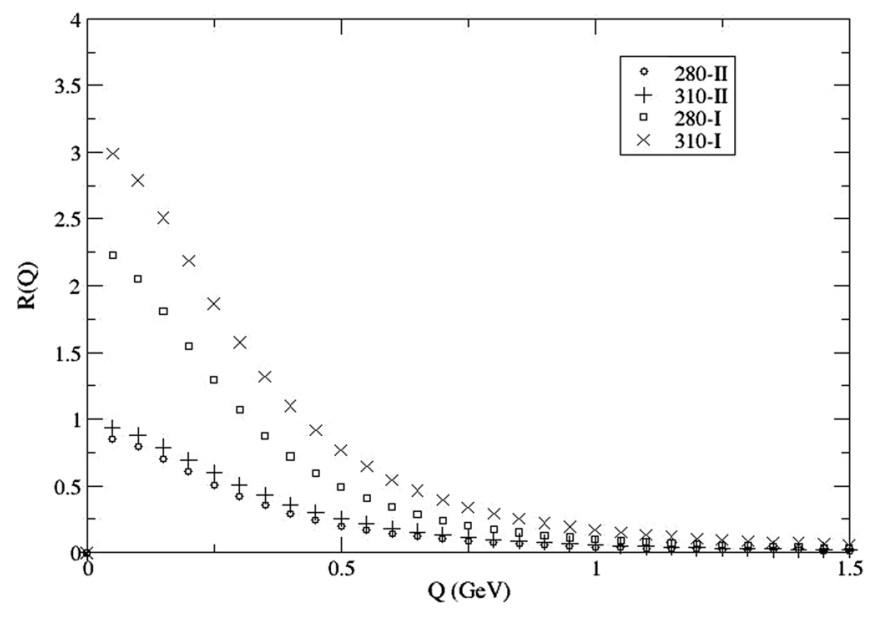

FIG. 10. The momentum-dependent deviation of the Goldberger-Treiman relation for the gluon propagators $D_{I}(k)$ and $D_{I I}(k)$ and quark effective masses $M^{*}=280$ and $310 \mathrm{MeV}$, by considering normalized definitions for $G_{A}^{U}(0,0)$ and $G_{\mathrm{ps}}^{U}(0,0)$ such as to satisfy the GTR at $Q=0$.

\section{Averaged quadratic radii}

Next, the corresponding strong averaged quadratic radii are defined from the different pion-constituent quark couplings presented above. Since the form factors are dimensionless the corresponding axial and pseudoscalar quadratic radii were defined by

$$
\begin{gathered}
\left\langle r^{2}\right\rangle_{A}^{W}=-\left.6 \frac{d G_{A}^{W}(0, Q)}{d Q^{2}}\right|_{Q=0}=\left\langle r^{2}\right\rangle_{V}^{W}, \\
\left\langle r^{2}\right\rangle_{A}^{W, \mathrm{tr}}=-\left.6 \frac{d G_{A}^{W, \mathrm{tr}}(0, Q)}{d Q^{2}}\right|_{Q=0}=\left\langle r^{2}\right\rangle_{V}^{W, \mathrm{tr}}, \\
\left\langle r^{2}\right\rangle_{A}^{U}=-\left.6 \frac{d G_{A}^{U}(0, Q)}{d Q^{2}}\right|_{Q=0}=\left\langle r^{2}\right\rangle_{V}^{U}, \\
\left\langle r^{2}\right\rangle_{\mathrm{ps}}=-\left.6 \frac{d G_{\mathrm{ps}}^{U}(0, Q)}{d Q^{2}}\right|_{Q=0}=\left\langle r^{2}\right\rangle_{2 j s}, \\
\left\langle r^{2}\right\rangle_{\mathrm{ps}}^{\mathrm{tr}}=-\left.6 \frac{d G_{\mathrm{ps}}^{U, \mathrm{tr}}(0, Q)}{d Q^{2}}\right|_{Q=0}=\left\langle r^{2}\right\rangle_{2 j s}^{\mathrm{tr}} .
\end{gathered}
$$

where in the right hand side of these expressions the relations to vector and scalar quadratic radii from the form factors defined in the previous sections are exhibited. In [28], the light vector/axial meson couplings to constituent quarks were considered to provide corresponding quadratic radii. The corresponding averaged axial and vector quadratic radii seen by the coupling to the pion, presented in this work, also turn out to be equal. Both results, from the pion and axial mesons couplings, are to be added, i.e., in fact expressions (39)-(41) provide corrections to the corresponding quadratic radii. However, their experimental

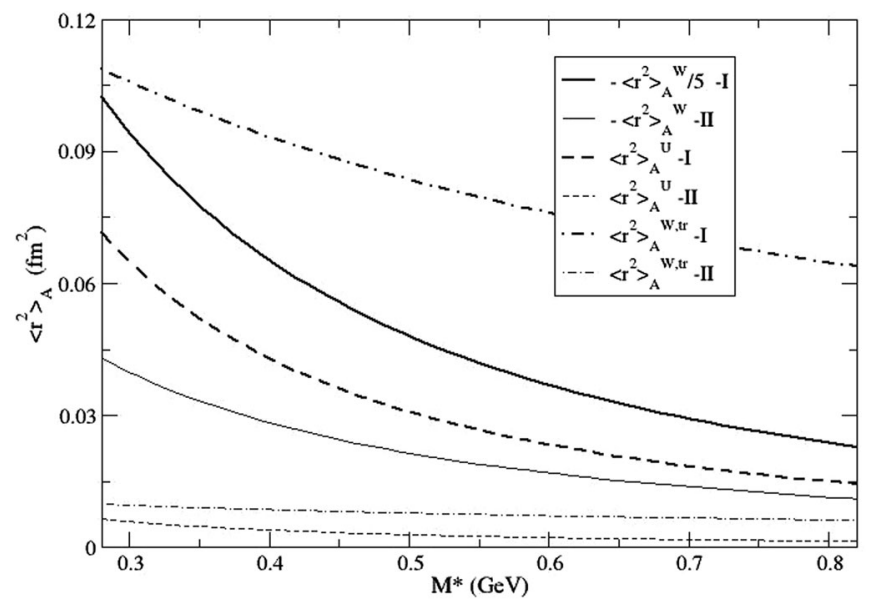

FIG. 11. The axial quadratic averaged radius (contribution) for the two pion definitions, $W$ and $U$, and two gluon propagators, $I$ and $I I$, as functions of the effective quark mass $M^{*}$. The factors $h_{a}$ were chosen to be $h_{I}=3$ and $h_{I I}=1$. The numerical result for $\left\langle r^{2}\right\rangle_{A}^{W}$ has a sign minus and the results for the gluon propagator $D_{I}$ it is divided by 5 to fit in the scale of the figure.

values, at the nucleon level, must receive further corrections since vector and axial a.q.r. are different from each other and expected to follow $\sqrt{\left\langle r_{V}^{2}\right\rangle /\left\langle r_{A}^{2}\right\rangle} \simeq 1.6$ [12].

In Fig. 11, the different estimations for the axial quadratic radius contribution for the two pion definitions, $W$ and $U$, and for the two gluon propagators, as functions of the quark effective mass $M^{*}$. In the figures with a.q.r., the factors $h_{a}$ were considered $h_{I}=3$ and $h_{I I}=1$, such that results could be compared with results from [28]. In the case of the Weinberg definition, there are also results for the truncated expression. The axial radius (contribution) $\left\langle r^{2}\right\rangle_{A}^{W}$ is negative because of the behavior of the axial form factor close to zero exchanged momentum and this unexpected behavior is corrected by the truncated expression as discussed above. Besides the problem with the sign for $\left\langle r^{2}\right\rangle_{A}^{W}$ it is also noted a different behavior in the $M^{*}$ dependence of the axial quadratic radii between $\left\langle r^{2}\right\rangle_{A}^{W}$ and $\left\langle r^{2}\right\rangle_{A}^{W, \text { tr }}$, being that the former presents a stronger variation for increasing $M^{*}$ and the latter a smoother variation.

These axial quadratic radii correction due to the pion are smaller than the vector/axial quadratic radii due to the vector/axial light mesons calculated with the same method for both gluon propagators in [28]. In that work, the axial quadratic radii found from the coupling to the $A_{1}$ meson, $\left\langle r_{\text {a.m. }}^{2}\right\rangle_{A}$, were estimated to be in the following range of values - for the same range of values of the quark effective mass $M^{*}$-by keeping the corresponding $h_{a}$ to the ones used in the figures for the a.q.r.:

$$
\begin{array}{ll}
\left\langle r_{\text {a.m. }}^{2}\right\rangle_{A} \sim 0.4-0.2 \mathrm{fm}^{2}, & D_{I I}(k), \\
\left\langle r_{\text {a.m. }}^{2}\right\rangle_{A} \sim 4.0-2.0 \mathrm{fm}^{2}, & D_{I}(k),
\end{array}
$$




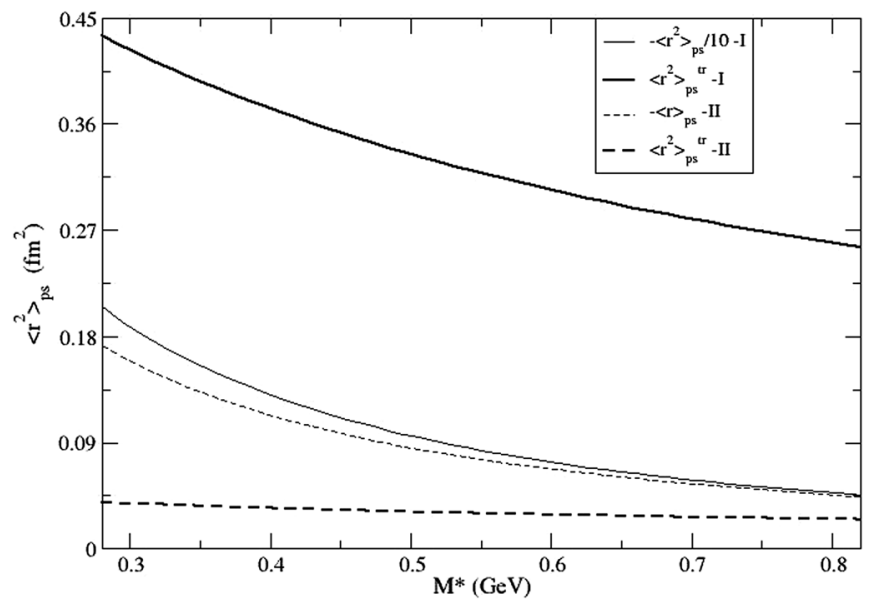

FIG. 12. The pseudoscalar averaged quadratic radius and two gluon propagators, $D_{I}$ and $D_{I I}$, as functions of the effective quark mass $M^{*}$, with factors $h_{a}$ from the previous figures. The numerical result for $\left\langle r^{2}\right\rangle_{\mathrm{ps}}$ has a sign minus and the results for the gluon propagator $D_{I}$ it is divided by 10 to fit in the scale of the figure. The factors $h_{a}$ were chosen to be $h_{I}=3$ and $h_{I I}=1$.

respectively, for gluon propagators $D_{I I}(k)$ and $D_{I}(k)$. Of course, the estimations for $\left\langle r_{\mathrm{a} . \mathrm{m} .}^{2}\right\rangle_{A}$ with $D_{I}(k)$ are extremely large, also present in Figs. 11 and 12, and this is attributed to the corresponding quark-gluon coupling constant and gluon propagator strengths. Both resulting values, however, are basically of the order of magnitude as (or larger than) the estimation for constituent quark radius $\sqrt{\left\langle r^{2}\right\rangle_{C Q}} \simeq 0.2-0.3 \mathrm{fm}[12,24]$, apart from normalizations of the quark-gluon coupling constant. The experimental value for the axial radius of the nucleon is $\left\langle r_{A}^{2}\right\rangle^{1 / 2} \simeq$ $0.68 \mathrm{fm}[1,13]$ and there are many estimations from lattice $\left\langle r_{A}^{2}\right\rangle^{1 / 2} \simeq 0.45-0.50 \mathrm{fm}$, for example in $[18,46]$ and references therein.

Similar behavior was found for the pseudoscalar quadratic radii presented in the next Fig. 12 from expressions (42), (43), complete and truncated ones, as functions of the quark effective mass $M^{*}$ for the two gluon propagators. The nontruncated expression provides negative values and they are presented with a sign minus. One of them is divided by factor 10 for $D_{I}(k)$ to fit into a reasonable scale of the figure. To make possible a correct calculation with the previous figure it was assumed $h_{I}=3$ and $h_{I I}=1$. The axial $\left\langle r^{2}\right\rangle_{A}$ contribution was found to be smaller than the pseudoscalar $\left\langle r^{2}\right\rangle_{\mathrm{ps}}$ in all cases. This is related to the fact that the pseudoscalar form factor normalization is larger than the axial form factor one. At this level, all the form factors reduce to only $F_{1}(K, Q)$ and $F_{2}(K, Q)$ and the truncated version $F_{1}^{\operatorname{tr}}(K, Q)$. However, the difficulty in fixing the quark-gluon vertex and the overall momentum behavior of the quark and gluon propagators cannot be neglected. When compared to the value $\sqrt{\left\langle r^{2}\right\rangle_{C Q}} \simeq 0.2-0.3 \mathrm{fm}$ from [12,24], the gluon propagator $D_{I}(k)$ provides larger values for $\left\langle r^{2}\right\rangle$ and the gluon propagator $D_{I I}(k)$ again provides smaller values. The reasons for the differences between $\left\langle r^{2}\right\rangle_{\mathrm{ps}}$ and the truncated- $\left\langle r^{2}\right\rangle_{\text {ps }}$ must be the same as the ones responsible for the discrepancies in the axial radii from Fig. 11. Besides that, it might be interesting, for the sake of comparison, to compare with the scalar radius of the lightest hadron, the pion, that has been calculated, for example, in lattice with $\left\langle r^{2}\right\rangle_{s}=0.6 \mathrm{fm}^{2}$ [47]. The pion charge radius has estimations for example in lattice $\left\langle r^{2}\right\rangle=0.37 \mathrm{fm}^{2}$ [8] and with $\operatorname{SDE}\left\langle r^{2}\right\rangle=0.46-0.48 \mathrm{fm}^{2}$ [48], whereas its experimental value $\left\langle r^{2}\right\rangle \simeq 0.45 \mathrm{fm}^{2}[9,20]$. The pion scalar radius seems, therefore, to be larger than its charge radius analogously to the fact that according to the present results the pseudoscalar, and also scalar, radii are larger than the axial and vector radii.

\section{SUMMARY AND DISCUSSION}

Pion-constituent quark momentum-dependent form factors were investigated from the one-loop background field method for the one nonperturbative gluon exchange quark interaction from the QCD effective action. At this level, the pseudoscalar coupling only shows up for the usual pion field definition in terms of unitary functions $U, U^{\dagger}$ but not for the Weinberg pion field. Besides the usual pseudoscalar pion coupling, other derivative pion -scalar and pseudoscalar currents form factors were also found in the leading order of the determinant expansion in expressions (18), (15) and also (19) and (22). Several of them have a reduced strength with respect to the usual scalar and pseudoscalar form factors by a constant coefficient of the order of $1 / M^{*}$. By means of an integration by parts these terms might contribute for the vector and axial channels. All the (eleven) resulting form factors, pseudoscalar, scalar, vector and axial, were found to be written in terms of only two momentum-dependent functions $F_{1}(0, Q)$ and $F_{2}(0, Q)$ for zero external constituent quark momentum, with different coefficients. A truncated momentum dependence of the quark kernel for $F_{1}(0, Q)$ was also considered such that the resulting form factors, $G_{A}^{W, \text { tr }}(0, Q)$ and $G_{\mathrm{ps}}^{U, \text { tr }}(0, Q)$, were shown to have a decreasing monotonic behavior more similar to the experimental results, corresponding rather to the function $F_{2}(0, Q)$. The truncated expressions might in fact correspond to considering running momentumdependent effective sea quark mass from the gap equation. Besides that, these truncated expressions yield positive quadratic averaged radii. Different values for the sea quark effective mass $M^{*}$ were considered and it mostly contributes for the overall normalization of the form factors. The first momentum-dependent function presented was the constituent quark effective mass correction $M_{3}(Q)$. Its momentum dependence is in excellent agreement with estimations from SDE calculations, except for its overall normalization that appeared to be very large due to absence 
of an UV cutoff. It is important to stress that the mechanisms that give rise to the gap effective mass $M^{*}$ and to the mass $M_{3}(Q)$ are different. However, the behavior of constituent quark mass $M_{3}(Q)$ is nearly independent of the scalar condensate contribution for the (constant) quark effective mass $M^{*}$. At the level of the calculation presented, the axial and vector form factors are equal to each other for each of the pion field definitions. The same chiral relation appeared for the scalar and pseudoscalar form factors for the second pion definition. The axial and pseudoscalar form factors were compared to fittings of available experimental data for pion nucleon form factors by adjusting the values at zero momenta. Results showed that the momentum dependence of constituent quark coupling to pions is not very different from the nucleon coupling to pions. The larger difference between experimental (nucleon form factor) values and the present form factors appear in the range of $0.15<Q<1.4 \mathrm{GeV}$ for $M^{*}=0.31 \mathrm{GeV}$. This might signal the need for improved momentum structure of the quark and gluon kernels but it might also signal need to account for effects from nucleon structure. The pseudoscalar form factor has a larger strength than the axial one, in agreement with expectations from phenomenology. This conclusion remains valid if other components for the axial form factor are included such as the coupling to light axial mesons, as seen by comparing with results from Ref. [28] in which vector/axial mesons couplings to constituent quark had been investigated by means of the same method employed in the present work. A systematic and more general analysis will be presented elsewhere. The pseudoscalar form factor at the spacelike point $Q^{2}=-m_{\pi}^{2}$, closer to current physical definitions of $g_{\pi N}$, was obtained for the complete (or truncated) expressions being smaller (or larger) than the zero momentum $Q^{2}=0$ case. Different momentum-dependent and -independent ratios between the form factors were also presented. Some of them simply show the resulting chiral symmetry relations, e.g., between vector and axial ones, or between scalar and pseudoscalar ones. The momentum dependence of the GoldbergerTreiman relation (GTR) was also presented by considering the pseudoscalar and axial form factors for timelike momenta and a qualitative agreement with calculations at the nucleon level as found. Finally the corresponding results for the pseudoscalar and contribution to the axial constituent quark averaged quadratic radii were obtained as functions of a constant quark effective mass $M^{*}$ from the gap equation. In particular, resulting values for the axial/ vector quadratic radii are somewhat smaller than estimations of the constituent quark axial/vector radii from the coupling to light axial/vector mesons obtained with the same method [28]. The structureless pion limit might have had effect on the estimations but this structureless limit had also been considered for the vector/axial mesons. In general, the pseudoscalar quadratic radius is larger than the axial radius (from both couplings to pions and axial mesons) due to the corresponding form factors normalizations. This becomes clear by noting all the quadratic radii and form factors depend on only two momentum-dependent functions. The relevance of each of the constituent quark d.o.f. presented in this work and [28] for nucleon structure and corresponding form factors is to be investigated elsewhere.

\section{ACKNOWLEDGMENTS}

The author thanks P. Bedaque, G. I. Krein, and G. Eichmann for brief discussions, and C.D. Roberts for kindly sending numerical results for the running quark mass from SDE from Ref. [44] plotted in Fig. 3. The author also acknowledges participation in the project Instituto Nacional de Ciência e Tecnologia-Física Nuclear e Aplicações (INCT-FNA), Proc. No. 464898/2014-5.
[1] K. L. Miller et al., Study of the reaction $\nu_{\mu} d \rightarrow \mu^{-} p p_{s}$, Phys. Rev. D 26, 537 (1982); T. Kitagaki et al., High-energy quasielastic $\nu_{\mu} n \rightarrow \mu^{-} p$ scattering in deuterium, Phys. Rev. D 28, 436 (1983).

[2] J.-M. Gaillard and G. Sauvage, Hyperon beta decays, Annu. Rev. Nucl. Part. Sci. 34, 351 (1984).

[3] S. Choi et al., Axial and Pseudoscalar Nucleon Form Factors from Low Energy Pion Electroproduction, Phys. Rev. Lett. 71, 3927 (1993).

[4] G. Bardin, J. Duclos, A. Magnon, J. Martino, A. Richter, E. Zavattini, A. Bertin, M. Piccinini, and A. Vitale, Measurement of the ortho para transition rate in the $p_{p}^{\mu}$ molecule and deduction of the pseudoscalar coupling constant $g_{p}^{\mu}$, Phys. Lett. 104B, 320 (1981).
[5] V. A. Andreev et al. (MuCap Collaboration), Measurement of the Muon Capture Rate in Hydrogen Gas and Determination of the Protons Pseudoscalar Coupling $g_{P}$, Phys. Rev. Lett. 99, 032002 (2007).

[6] E. J. Beise, The axial form factor of the nucleon, Eur. Phys. J. A 24, 43 (2005).

[7] P. Maris and C. D. Roberts, Dyson-Schwinger equations: A tool for hadron physics, Int. J. Mod. Phys. E 12, 297 (2003); P. Tandy, Hadron physics from the global color model of QCD, Prog. Part. Nucl. Phys. 39, 117 (1997).

[8] J. van der Heide, J.H. Koch, and E. Laermann, Pion structure from improved lattice QCD: Form factor and charge radius at low masses, Phys. Rev. D 69, 094511 (2004). 
[9] D. Drechsel and Th. Walcher, Hadron structure at low $\mathrm{Q}^{2}$, Rev. Mod. Phys. 80, 731 (2008).

[10] T. Yamazaki, Y. Aoki, T. Blum, H.-W. Lin, S. Ohta, S. Sasaki, R. Tweedie, and J. Zanotti, Nucleon form factors with $2+1$ flavor dynamical domain-wall fermions, Phys. Rev. D 79, 114505 (2009).

[11] M. Constantinou, Hadron structure, Proc. Sci. LATTICE2014 (2015) 001 [arXiv:1411.0078].

[12] U. Vogl and W. Weise, The Nambu and Jona-Lasinio model: Its implications for hadrons and nuclei, Prog. Part. Nucl. Phys. 27, 195 (1991).

[13] M. Hoferichter, C. Ditsche, B. Kubis, and U.-G. Meissner, Dispersive analysis of the scalar form factor of the nucleon, J. High Energy Phys. 06 (2012) 063.

[14] G. Ramalho and K. Tsushima, Holographic estimate of the meson cloud contribution to nucleon axial form factor, Phys. Rev. D 94, 014001 (2016).

[15] G. Eichmann and C. S. Fischer, Nucleon axial and pseudoscalar form factors from the covariant Faddeev equation, Eur. Phys. J. A 48, 9 (2012).

[16] V. Bernard, L. Elouadrihiri, and Ulf-G. Meissner, Axial structure of the nucleon, J. Phys. G 28, R1 (2002).

[17] J. D. Bratt et al., Nucleon structure from mixed action calculations using $2+1$ flavors of asqtad sea and domain wall valence fermions, Phys. Rev. D 82, 094502 (2010).

[18] C. Alexandrou, M. Brinet, J. Carbonell, M. Constantinou, P. A. Harraud, P. Guichon, K. Jansen, T. Korzec, and M. Papinutto, Axial nucleon form factors from lattice QCD, Phys. Rev. D 83, 045010 (2011); C. Alexandrou, M. Constantinou, K. Hadjiyiannakou, K. Jansen, C. Kallidonis, G. Koutsou, and A. Vaquero Aviles-Casco, Nucleon axial form factors using lattice QCD simulations with a physical value of the pion mass, Phys. Rev. D 96, 054507 (2017).

[19] A. Beyer et al., The Rydberg constant and proton size from atomic hydrogen, Science 358, 79 (2017).

[20] C. Patrignani et al. (Particle Data Group), Review of particle physics, Chin. J. Phys. C 40, 100001 (2016).

[21] S. Weinberg, Pions in Large N Quantum Chromodynamics, Phys. Rev. Lett. 105, 261601 (2010).

[22] M. Lavelle and D. McMullan, Constituent quarks from QCD, Phys. Rep. 279, 1 (1997); E. de Rafael, The constituent Chiral quark model revisited, Phys. Lett. B 703, 60 (2011).

[23] A. W. Thomas, Chiral extrapolation of hadronic observables, Nucl. Phys. B, Proc. Suppl. 119, 50 (2003); R. D. Young, D. B. Leinweber, and A. W. Thomas, Convergence of chiral effective field theory, Prog. Part. Nucl. Phys. 50, 399 (2003), and references therein.

[24] R. Petronzio, S. Simula, and G. Ricco, Possible evidence of extended objects inside the proton, Phys. Rev. D 67, 094004 (2003); Erratum, Phys. Rev. D 68, 099901(E) (2003).

[25] F. L. Braghin, Quark and pion effective couplings from polarization effects, Eur. Phys. J. A 52, 134 (2016).

[26] F. L. Braghin, Low energy constituent quark and pion effective couplings in a weak external magnetic field, Eur. Phys. J. A 54, 45 (2018).
[27] C. Downum, T. Barnes, J. R. Stone, and E. S. Swanson, Nucleon-meson coupling constants and form factors in the quark model, Phys. Lett. B 638, 455 (2006).

[28] F. L. Braghin, Light vector and axial mesons effective couplings to constituent quarks, Phys. Rev. D 97, 054025 (2018).

[29] F. L. Braghin, Constituent quark-light vector mesons effective couplings in a weak background magnetic field, Phys. Rev. D 97, 014022 (2018).

[30] Y. B. Yang, J. Liang, Yu-J. Bi, Y. Chen, T. Draper, K. F. Liu, and Z. Liu, Proton Mass Decomposition from the QCD Energy Momentum Tensor, Phys. Rev. Lett. 121, 212001 (2018), and references therein.

[31] J. M. Cornwall, Entropy, confinement, and chiral symmetry breaking, Phys. Rev. D 83, 076001 (2011).

[32] P. Maris and P. C. Tandy, Bethe-Salpeter study of vector meson masses and decay constants, Phys. Rev. C 60, 055214 (1999).

[33] C. D. Roberts, R. T. Cahill, and J. Praschifka, The effective action for the goldstone modes in a global colour symmetry model of QCD, Ann. Phys. (N.Y.) 188, 20 (1988).

[34] D. Ebert, H. Reinhardt, and M. K. Volkov, Effective hadron theory of QCD, Prog. Part. Nucl. Phys. 33, 1 (1994).

[35] D. Binosi, L. Chang, J. Papavassiliou, and C. D. Roberts, Bridging a gap between continuum-QCD and ab initio predictions of hadron observables, Phys. Lett. B 742, 183 (2015), and references therein.

[36] K.-I. Kondo, Abelian-projected effective gauge theory of QCD with asymptotic freedom and quark confinement, Phys. Rev. D 57, 7467 (1998).

[37] K. Higashijima, Dynamical chiral-symmetry breaking, Phys. Rev. D 29, 1228 (1984).

[38] B. Holdom, Approaching low-energy QCD with a gauged, nonlocal, constituent-quark model, Phys. Rev. D 45, 2534 (1992).

[39] Q. Wang, Y.-P. Kuang, X.-L. Wang, and M. Xiao, Derivation of the effective chiral Lagrangian for pseudoscalar mesons from QCD, Phys. Rev. D 61, 054011 (2000); K. Ren, H.-F. Fu, and Q. Wang, Derivation of the effective chiral Lagrangian for pseudoscalar, scalar, vector, and axial-vector mesons from QCD, Phys. Rev. D 95, 074012 (2017).

[40] F. L. Braghin, SU(2) low energy quark effective couplings in weak external magnetic field, Phys. Rev. D 94, 074030 (2016).

[41] L. F. Abbott, Introduction to the background field method, Acta Phys. Pol. B 13, 33 (1982).

[42] S. Weinberg, The Quantum Theory of Fields (Cambridge University Press, Cambridge, England, 1996), Vol. 2.

[43] J. C. Bloch, C. D. Roberts, and S. M. Schmidt, Selected nucleon form factors and a composite scalar diquark, Phys. Rev. C 61, 065207 (2000).

[44] C. Chen, B. El-Bennich, C. D. Roberts, S. M. Schmidt, J. Segovia, and S. Wan, Structure of the nucleon's low-lying excitations, Phys. Rev. D 97, 034016 (2018).

[45] S. Sasaki and T. Yamazaki, Nucleon form factors from quenched lattice QCD with domain wall fermions, Phys. Rev. D 78, 014510 (2008). 
[46] R. Gupta, Y.-C. Jang, H.-W. Lin, B. Yoon, and T. Bhattacharya, Axial-vector form factors of the nucleon from lattice QCD, Phys. Rev. D 96, 114503 (2017); Y.-C. Jang, T. Bhattacharya, R. Gupta, H.-W. Lin, and B. Yoon, Nucleon axial and electromagnetic form factors, EPJ Web Conf. 175, 06033 (2018).
[47] V. Gulpers, G. von Hippel, and H. Wittig, The scalar radius of the pion from lattice QCD in the continuum limit, Eur. Phys. J. A 51, 158 (2015).

[48] P. Maris and P.C. Tandy, The quark-photon vertex and the pion charge radius, Phys. Rev. C 61, 045202 (2000). 